\title{
The Application of BIM Technology in Reconstruction of a Basement
}

\author{
Jia Guangyin"1, a, Hong Sen 1, b \\ 'Zaozhuang University, Zaozhuang 277160, China \\ ahappy_design@126.com,booglejob@126.com
}

Keywords: computer-aided design. modeling.

Abstract. With the continuous development of computer technology, computer-aided design software functions are increasingly perfect and convenient. BIM technology has obvious guiding advantages in the process of architectural design and construction. Based on a basement conversion process as an example in this paper, the core of the BIM modeling software Revit and Navisworks will be used to guide the construction of the project design.

\section{Introduction}

BIM is the full name of Building Information Modeling[1,2], whose technical concept runs through the program of scrutiny, pre-planning, drawing design, construction guidance and post-operation, management, maintenance. The subsequent AutoCAD and other related software of Autodesk Company will provide great convenience for the development of technology, especially in the process of construction drawings, which relied mainly on the manual drawing at the early time. However, the traditional drawing software is more demanding with the high efficiency, high quality requirements on the entire project process and the design requirements of complex abnormity structures by modern buildings. The emerging BIM-related software can be visualized at the design stage, designers can complete the architectural design effectively by using three-dimensional thinking, and at the same time the owners are always available to get project information, which greatly reduces the communication disorders between designers and owners[3]. Based on a basement conversion process as an example in this paper, the core of the BIM modeling software Revit and Navisworks will be used to guide the construction of the project design.

\section{Project Overview}

The construction site is the basement of a building which was originally an employee club. It has been abandoned as close to the building pump room, no normal lighting parts, and in damp environment for a long time. According to the requirements of the owner, it needs to be transformed into a casual cafe. In the process of transformation, in order to ensure the safety of the structure, changes will not be adopted in the structure. And for aesthetic needs, false windows, awning and other components need to be set up. We use Revit and Navisworks as the main tools to build three-dimensionally modeling and roaming to provide guidance for construction.

\section{The modeling process}

\section{1 model foundation}

The latest version of the Revit software has integrated three modules of Architecture, Structure and MEP and they are in good collaboration and share among each other. After opening revit software, the sample file needs to be selected in new project. It should be noted that the default suffix of the sample file is *.rte and the default suffix of the project file is *.rvt. After setting the basic drawing environment, you need to start drawing with the elevation and axis net. In the modeling process, the biggest problem is the production of complex family based on solid models, as shown in Fig.2. The two white columns in the middle is the structural column where the entity building itself exists. In order to minimize disturbance to the original building, a space-saving and beautiful bar is 
required to build surrounded by these two pillars. Here we use the family production. In view of the production of part of the family, we will introduce in Section 2.2. Fig.1 shows the three-dimensional model established by revit. In order to ensure that the bar can leave a comfortable operation space for staff during the construction after coming into use, it is necessary to control the exact size between the bar and the column. Fig. 2 is the actual construction drawings. Fig.3 is the realistic scene after completing the decoration and renovation part. It can be seen that the project design in the beginning and the construction in the later period as well as realistic image of high fidelity after completion of the project, which can be used to guide the actual construction.

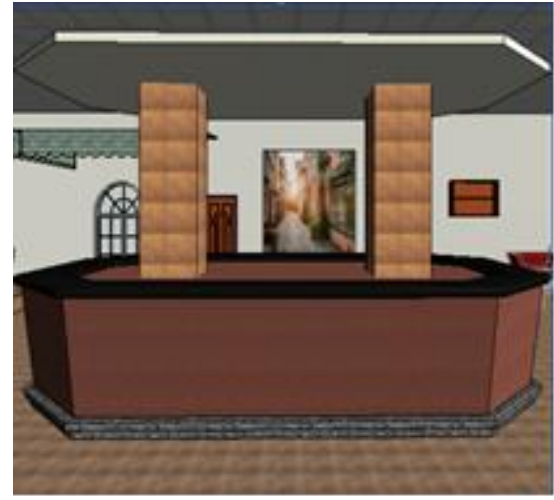

Fig.1

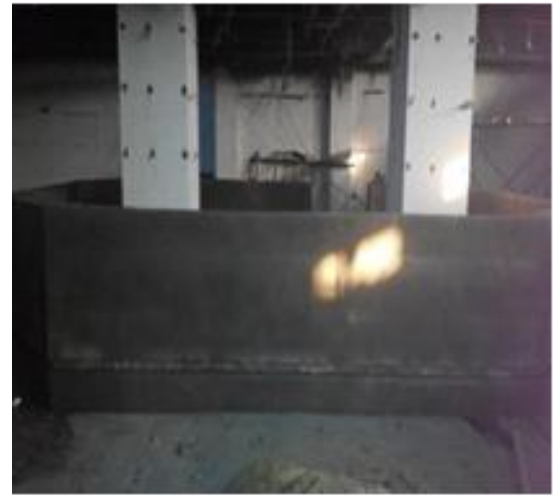

Fig.2

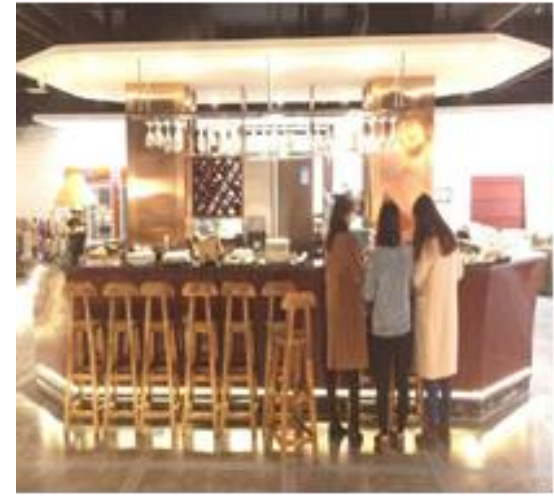

Fig.3

We take screenshots of three different periods of time from another angle for contrast. The 3D model is shown in Fig.4 created by revit. For the requirement of the scene, the bland window is set in the wall and illumination can be simulated through the lights in the back. The window is located in the wall, which can be observed in Fig. 5. In order to increase the romantic atmosphere of the cafe, sun shading canopy is set. The realistic image of the project is shown in Fig. 6. The specific production of the sun shading canopy will be discussed in detail in section 2.2.

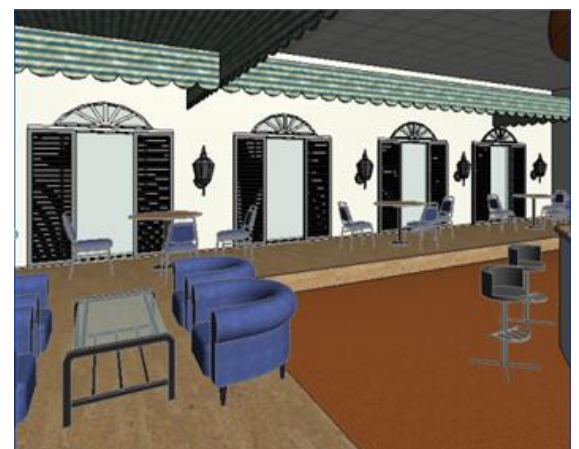

Fig.4

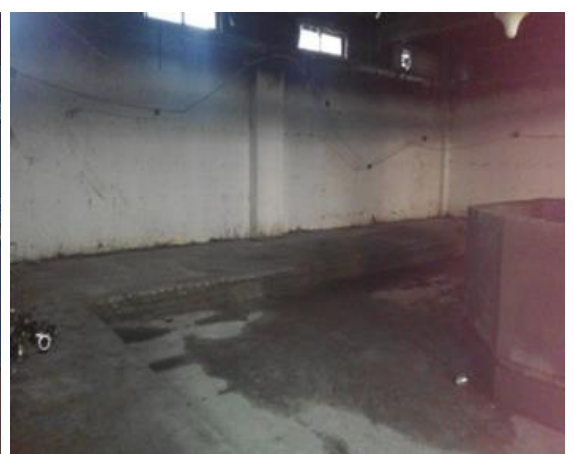

Fig.5

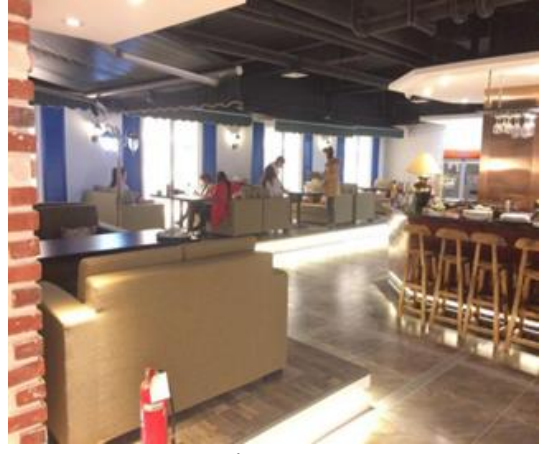

Fig.6

\subsection{Production of complex families}

According to the project requirements, a canopy needs to be created to increase the site of the real atmosphere. The production of the canopy is related to a very core function of the revit, which is the production of the family. The whole production process of the canopy will be introduced briefly in the following to elaborate the family production.

The whole process is based on the standard regular profile family to build rules family. As shown in Fig.7, two-dimensional contour drawing is first performed by the stretch command. On the basis of Fig.7, a 3D solid shape is created by parametric solid extrusion, whose effect is shown in Fig. 8. As shown in Fig.9, the design of the canopy end is through the hollow stretch command, in the previously created three-dimensional solid model of the components of the outline of its outline shape. Tensile shear is performed based on the completed contour lines, and the effect is shown in Fig.10. Then, we need to define the intrinsic parameters of components to facilitate the coordination 
of the design of the later changes, based on the design of the actual project components to give the real material, through Autodesk Revit material library selection, select the appearance of the "fabric" Cloth - blue and white "material. The final real effect of the canopy is completed.

Fig.7

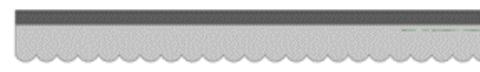

Fig.10

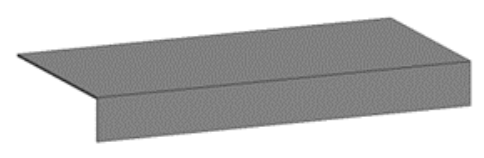

Fig.8

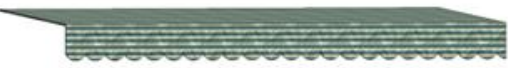

Fig.11

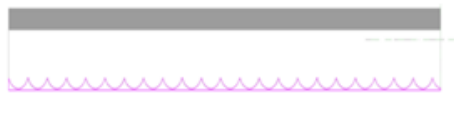

Fig.9

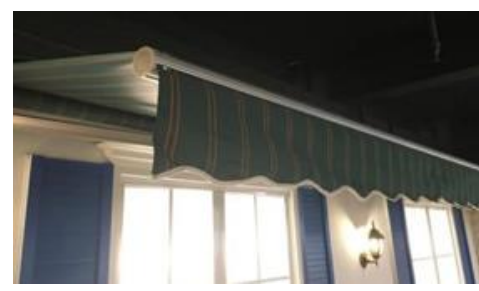

Fig.12

\subsection{Collision Check}

Autodesk Navisworks software works very well with Revit, and the model we created in the previous version was imported into Navisworks for roaming collision checking, which is able to visualize roaming, collision checking of the design models that have been created, create a realistic real-life experience before the project is actually completed and can identify issues that may present in the design, such as collisions of pipes, collision of beams and doors, and lack of reserve height.

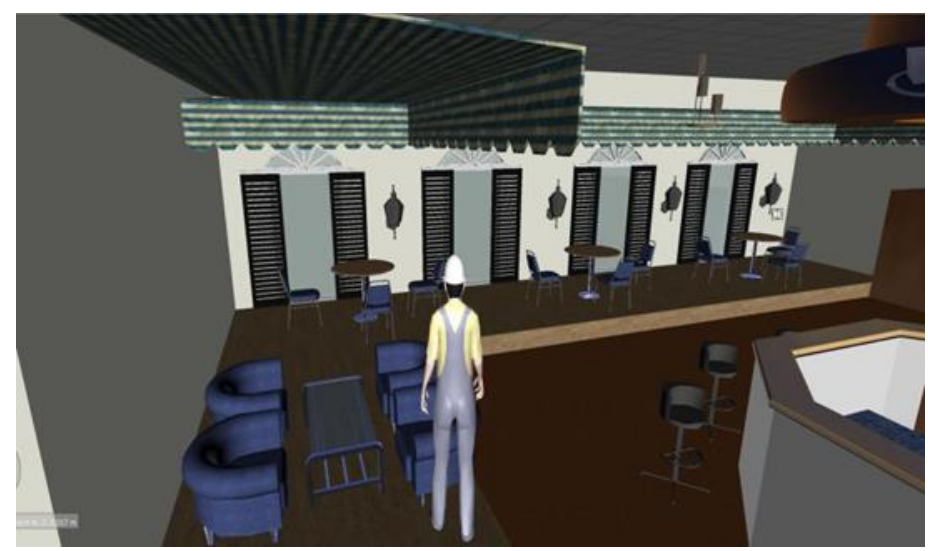

Fig.13

With the continuous development of computer technology, computer-aided design software functions are increasingly perfect and convenient. BIM technology has obvious guiding advantages in the process of architectural design and construction.

\section{References}

[1] Wang Danjing. Bim technology and the influence of Bim skills contest for construction engineering group construction[J]. Journal of Jiamusi Vocational Institute,2014,(11):192-193+198. 
[2] He Lingtong. Global BIM Application Status[J]. Construction Quality,2013,(03):12-19.

[3] Editorial Committee of BIM engineering and technical personnel professional skills training. The introduction of BIM Technology[M]. China Architecture\& Building Press,2016. 